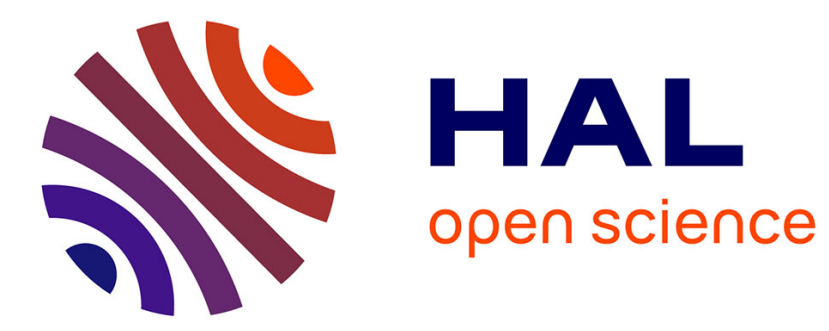

\title{
Harmonic balance/Galerkin method for non-smooth dynamic systems
}

\author{
W.-J. Kim, N. C. Perkins
}

\section{To cite this version:}

W.-J. Kim, N. C. Perkins. Harmonic balance/Galerkin method for non-smooth dynamic systems. Journal of Sound and Vibration, 2003, 261 (2), pp.213-224. 10.1016/S0022-460X(02)00949-5 . hal01693093

\section{HAL Id: hal-01693093 \\ https://hal.science/hal-01693093}

Submitted on 25 Jan 2018

HAL is a multi-disciplinary open access archive for the deposit and dissemination of scientific research documents, whether they are published or not. The documents may come from teaching and research institutions in France or abroad, or from public or private research centers.
L'archive ouverte pluridisciplinaire HAL, est destinée au dépôt et à la diffusion de documents scientifiques de niveau recherche, publiés ou non, émanant des établissements d'enseignement et de recherche français ou étrangers, des laboratoires publics ou privés. 


\title{
Harmonic balance/Galerkin method for non-smooth dynamic systems
}

\author{
W.- J. Kim, N.C. Perkins* \\ Department of Mechanical Engineering, University of Michigan, 2250 G. G. Brown, Ann Arbor, MI 48109, USA
}

Models of non- linear systems frequently introduce forces with bounded continuity resulting in nonsmooth (even discontinuous) flow. Examples include systems with clearances, backlash, friction, and impulses. Asymptotic methods require smooth (differentiable) flow and are therefore ill- suited for analyzing non-smooth systems. In these cases, the traditional harmonic balance method may be used to obtain approximate periodic solutions, but the method suffers from extremely slow convergence in general. Generalizations of the traditional harmonic balance method are introduced in this paper that result in superior convergence rates and superior modes of convergence. These improvements derive from the introduction of one or more expansion functions that possesses the same degree of continuity as the exact solution. In particular, forming an infinite series of such functions results in an expansion in the same function space of the exact solution. This expansion converges pointwise to the exact solution and to all derivatives thereof. These improvements are illustrated by example upon re-evaluating a classical single degree- of-freedom model for friction-induced vibration.

\section{Introduction}

Non-linear behavior is pervasive in mechanical systems and may arise from forces contributing to system stiffness, damping, and inertia. While many classes of non-linearities are described in the literature [1], a distinction that is central to this paper is that between smooth versus nonsmooth non-linearities; see, for example, Refs. [2-4]. A non-linearity will be considered smooth if it is a continuous function of the displacement field and all derivatives thereof. Examples of

\footnotetext{
*Corresponding author.

E-mail address: ncp@umich.edu (N.C. Perkins).
} 
smooth non-linearities include most material non-linearities and geometric non-linearities describing large amplitude motions. By contrast, non-smooth non-linearities produce forces that have bounded continuity and are therefore discontinuous functions of a derivative of the displacement field. Examples of non-smooth non-linearities include dry friction, impact, and ideal elastic-plastic material response.

The differentiability of smooth non-linearities renders them amenable to asymptotic methods including perturbation and averaging methods [1,5]. By contrast, the non-differentiability of nonsmooth non-linearities requires specialized methods of analysis. For instance, Pilipchuk [2,3] developed a novel non-smooth transformation for periodic motions using a pair of saw-tooth sine and rectangular cosine functions. The transformation eliminates discontinuities and leads to a smooth system that can be then evaluated using asymptotic methods. Similarly, Liu [6] decomposes a discontinuous periodic solution $x(t)=x_{1}(t)+H(t-a) x_{2}(t)$ into two smooth components $x_{1}(t)$ and $x_{2}(t)$ that are separated by a discontinuity at time $t=a$. Doing so permits the subsequent use of perturbation methods for the solution of the components $x_{1}(t)$ and $x_{2}(t)$. Here, $H(t-a)$ is the Heaviside function for a discontinuity at $t=a$.

Alternatively, one may also apply the harmonic balance method as the means to compute approximate periodic motions for systems with non-smooth non-linearities. In traditional harmonic balance [7], one constructs an approximate solution as a Fourier series of harmonic functions to represent a periodic response. The harmonic functions are infinitely differentiable. ${ }^{1}$ For systems with non-smooth non-linearities, a periodic motion will, in general, have bounded continuity. For instance, for a system with dry friction, a periodic motion that includes the stickslip transition will be continuous in both displacement and velocity, yet discontinuous in acceleration (a $\mathbf{C}^{1}$ function). Thus, the exact solution will not lie in the function space spanned by the terms of the harmonic balance series (the space of $\mathbf{C}^{\infty}$ functions). Moreover, the harmonic balance series for the acceleration will only converge in a mean square sense to the exact solution and not pointwise. In addition, the discontinuities in the acceleration will generate oscillations in the harmonic balance solution as a result of the Gibbs phenomenon [8]. The Gibbs phenomenon reduces the convergence rate to order $O(1)$ in the neighborhood of the discontinuity [9]. These effects conspire to produce extremely slow convergence for the harmonic balance series for nonsmooth dynamical systems.

The slow convergence of a harmonic balance solution for initial-value problems is analogous to the slow convergence of Ritz series observed in certain boundary-value problems. For instance, eigenvalue problems in linear structural dynamics can lead to non-smooth eigensolutions (in space) as a result of forces/moments produced by discrete elements such as springs (linear or rotational) [10], attached masses [11], cracks [12], etc. For example, the slow convergence of a Ritz series was noted in Ref. [10] for the case of a simply supported Euler-Bernoulli beam with a rotational spring in the interior. Accelerated convergence was achieved by adding the so-called forced modes to the Ritz series. Though not mentioned in Ref. [10], the first forced mode employed therein lies in the function space of the exact solution (a beam with an internal rotational spring will have mode shapes that are $\mathbf{C}^{1}$ in general as the spring produces a discontinuity in the internal

\footnotetext{
${ }^{1}$ Let $\mathbf{C}^{n}$ denote the space of functions possessing continuous derivatives through order $n$. Thus, the harmonic functions are elements of $\mathbf{C}^{\infty}$.
} 


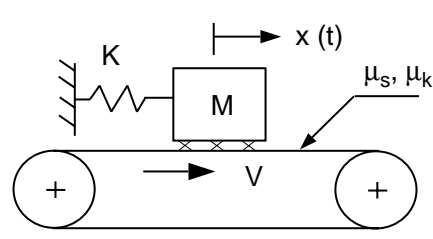

(a)

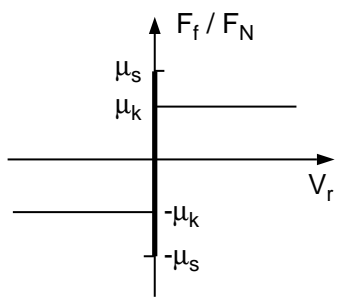

(b)

Fig. 1. (a) Classical single d.o.f. model for friction-induced vibration. (b) Coulomb friction law.

moment). It is this first forced mode term that significantly improves the convergence rate of the Ritz series. $^{2}$

The purpose of this paper is to introduce generalizations of the harmonic balance (or Galerkin method) that accelerate convergence relative to traditional harmonic balance for non-smooth dynamical systems. The methods are developed herein using the example of a single degreeof-freedom (d.o.f.) oscillator that exhibits stick-slip response due to dry friction. This model is presented in Section 2. In Section 3, we develop three approximations to the periodic motion of this oscillator using traditional harmonic balance and then two generalizations of this method that incorporate one or more $\mathbf{C}^{1}$ functions. The exact periodic solution to this non-smooth system is critically compared with the approximate solutions in Section 4. Conclusions regarding the convergence rate and convergence modes of all series are highlighted by this simple example.

\section{Non-smooth stick-slip model}

The schematic in Fig. 1. illustrates a classical single d.o.f. model used to describe frictioninduced vibrations [13]. The particle, in contact with the moving belt, experiences self-sustained stick-slip vibrations induced by dry friction. Such friction-induced vibrations arise in many applications including, for example, brake squeal, machine tool chatter, bowed string instruments, drying windshield wiper blades, etc; refer to reviews [14-16]. For instance, the oscillator in Fig. 1 may represent the first mode of a violin string for which the drawn bow is represented by the belt travelling with constant speed $V$.

A Coulomb friction law will be adopted for this model that incorporates classical static $\mu_{s}$ and kinetic $\mu_{k}$ friction coefficients; see Fig. 1(b). The equation of motion for this oscillator is given by

$$
\ddot{x}+\omega_{n}^{2} x=\frac{F_{f}\left(V_{r}\right)}{M},
$$

\footnotetext{
${ }^{2}$ It is curious that additional forced mode terms are also considered in Ref. [10] that have increasingly greater degrees of continuity and therefore do not lie in the function space of the exact solution. These additional terms have far less influence in the convergence rate relative to the first forced mode term as seen in the results in Ref. [10].
} 
where $\omega_{n}=\sqrt{K / M}$ denotes the natural frequency of the system. The friction force in Eq. (1) is specified as

$$
F_{f}=\left\{\begin{array}{lll}
K x & \text { for } V_{r}=0 \text { and }|K x| \leqslant \mu_{s} F_{N} & \rightarrow \text { stick, } \\
-\mu_{k} F_{N} \operatorname{sign}\left(V_{r}\right) & \text { for } V_{r} \neq 0 & \rightarrow \text { slip },
\end{array}\right.
$$

where $F_{N}$ is the normal force and $V_{r}=(\dot{x}-V)$ represents the relative velocity between the particle and the belt. The dynamics of this simple model has been extensively investigated; see, for instance, Refs. [13,15-18].

The sustained (friction-induced) vibration develops as follows. When $\dot{x}$ equals $V$, the particle sticks to the belt and is displaced ultimately to the static friction limit $|K x|=\mu_{s} F_{N}$. At this limit, the particle begins to slide back to the neutral position. During the slip period, energy is dissipated due to the non-conservative work by friction. If the energy input during the sticking phase balances the energy dissipation during the sliding phase, a distinctive non-smooth periodic motion (limit cycle) results as illustrated in Fig. 2. Note that this periodic solution may be readily deduced using initial conditions $\left(x_{0}, \dot{x}_{0}\right)$ satisfying $\left|x_{0}\right| \leqslant \mu_{s} F_{N} / K$ and $\dot{x}_{0}=V$. Thus, the stick form $(\dot{x}=V)$ of the equation of motion governs first. The slip form of the equation of motion governs second starting at the transition $|K x|=\mu_{s} F_{N}$. Since the displacement and velocity of the particle are continuous, these continuity conditions are applied as matching conditions between the stick and slip motions in arriving at the exact solution.

Fig. 2(a) shows the limit cycle in the phase plane consisting of the stick phase $\dot{x}=V(A \rightarrow B)$ followed by the slip phase $(B \rightarrow A)$. The particle oscillates about the position $x_{s}=\mu_{k} F_{N} / K$ with period $T$ as shown in Fig. 2(b). The corresponding particle velocity and acceleration are shown in Fig. 2(c) and (d) respectively. Since the speed of the particle remains less than or equal to $V$ during the limit cycle, the friction force always acts in the direction of the belt velocity. Note that the displacement is continuous and smooth, the velocity is continuous but not smooth, and consequently the acceleration is discontinuous. The discontinuities in the acceleration occur at the

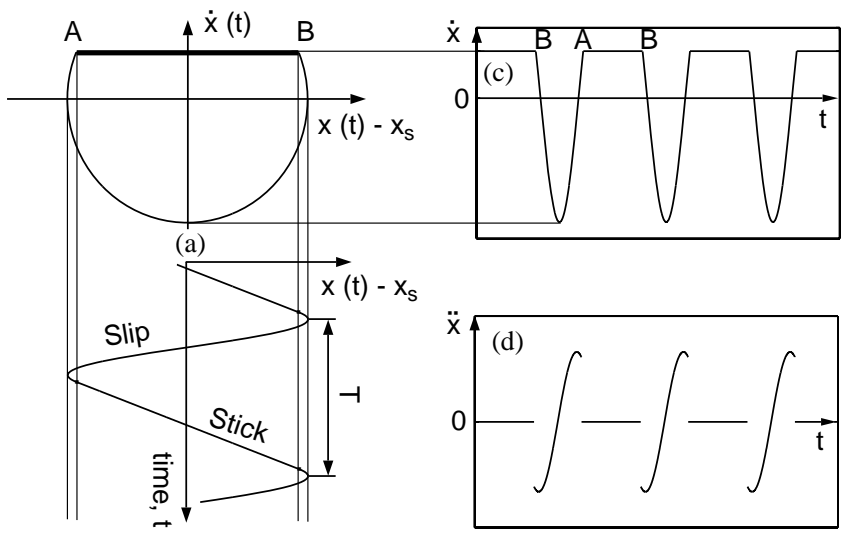

(b)

Fig. 2. Self-sustained oscillations: (a) limit cycle in phase plane, (b) corresponding displacement, (c) velocity, and (d) acceleration. 
transition times between stick and slip as dictated by the discontinuous friction force (2). Inspection of Eq. (2) readily demonstrates that the exact solution $x(t) \in \mathbf{C}^{1}$.

\section{Harmonic balance/Galerkin method}

Approximate solutions of Eq. (1) are now sought by using the harmonic balance or the Galerkin method [7]. Note that the term "Galerkin method" is most frequently associated with approximate solutions of boundary-value problems, while the term "harmonic balance" is most frequently associated with approximate periodic solutions of initial-value problems. However, the two methods represent the same strategy as they require the residual to be orthogonal to the expansion functions [7]. Hence, these terms will be used interchangeablely in this paper.

We shall now construct approximate periodic solutions to Eq. (1) using three variations of the harmonic balance/Galerkin method for this initial-value problem. We begin with the classical form of the harmonic balance method wherein the expansion functions are harmonics and are therefore $\mathbf{C}^{\infty}$. We shall then re-solve this problem upon adding a single (non-harmonic) expansion function that is $\mathbf{C}^{1}$ to the classical harmonic series. Finally, we shall construct an expansion using entirely $\mathbf{C}^{1}$ (non-harmonic) functions. The results of these three approximate series solutions will be critically compared in Section 4.

\subsection{Classical harmonic balance (Fourier series)}

The classical harmonic balance method begins by assuming a Fourier series expansion for periodic $x(t)$ given by

$$
x(t)=\frac{a_{0}}{2}+\sum_{n=1}^{N}\left[a_{n} \sin \left(n \omega_{0} t\right)+b_{n} \cos \left(n \omega_{0} t\right)\right] .
$$

The unknown quantities consist of the fundamental frequency $\omega_{0}$ which relates to the period $T$, the stick-to-slip transition time $t_{1}$, and the $2 N+1$ Fourier coefficients $a_{0}, a_{n}, b_{n}, n=1,2, \ldots, N$. Substitution of Eq. (3) into Eq. (1) results in a solution error or residual $R_{1}(t)$ given by

$$
R_{1}(t)=\frac{\omega_{n}^{2} a_{0}}{2}+\sum_{n=1}^{N}\left[\omega_{n}^{2}-\left(n \omega_{0}\right)^{2}\right]\left[a_{n} \sin \left(n \omega_{0} t\right)+b_{n} \cos \left(n \omega_{0} t\right)\right]-\frac{F_{f}}{M} .
$$

The Galerkin method requires that the residual $R_{1}$ be orthogonal to each expansion function over one (unknown) period $T=2 \pi / \omega_{0}$. Thus, the $2 N+1$ Fourier coefficients are found from the $2 N+1$ (harmonic balance) orthogonality conditions

$$
\begin{gathered}
\left\langle R_{1}(t), 1\right\rangle=0, \\
\left\langle R_{1}(t), \sin \left(j \omega_{0} t\right)\right\rangle=0, \quad j=1, \cdots, N, \\
\left\langle R_{1}(t), \cos \left(j \omega_{0} t\right)\right\rangle=0, \quad j=1, \cdots, N,
\end{gathered}
$$


where the inner product $\langle a(t), b(t)\rangle$ of two functions $a(t)$ and $b(t)$ is defined as

$$
\langle a(t), b(t)\rangle=\int_{0}^{t_{1}} a(t) b(t) \mathrm{d} t+\int_{t_{1}}^{T} a(t) b(t) \mathrm{d} t .
$$

We now introduce the initial conditions $\left(x_{0}, \dot{x}_{0}\right)$ that provide the two additional equations

$$
\begin{aligned}
& x(0)=\frac{a_{0}}{2}+\sum_{n=1}^{N} b_{n}=x_{0}, \\
& \dot{x}(0)=\sum_{n=1}^{N}\left(n \omega_{0}\right) a_{n}=\dot{x}_{0} .
\end{aligned}
$$

Eqs. (5)-(7), (9), and (10) provide $2 N+3$ non-linear algebraic equations for $2 N+3$ unknowns; namely, the unknown transition time $t_{1}$, the period $T\left(=2 \pi / \omega_{0}\right)$, and the Fourier coefficients $a_{0}, a_{n}, b_{n}, n=1,2, \ldots, N$. Solutions for these $2 N+3$ coupled equations are found numerically using multi-dimensional Newton-Raphson.

\subsection{Classical harmonic balance with one $\mathbf{C}^{1}$ term}

The classical harmonic balance solution above employs harmonic functions that are necessarily $\mathbf{C}^{\infty}$. This is at odds with the exact solution that is known (by inspecting of Eq. (1) above) to be $\mathbf{C}^{1}$. Consequently, the classical harmonic balance series cannot converge pointwise to $\ddot{x}$ and, in fact, converges only in the mean-square sense $\left(\mathbf{L}_{2}\right.$ norm). The resulting series converges slowly as will be seen in Section 4.

To accelerate the convergence rate, one additional term is added to Eq. (3) akin to the "forced modes" introduced in Ref. [10] for a linear eigenvalue problem. To this end, consider the new expansion

$$
x(t)=\frac{a_{0}}{2}+\sum_{n=1}^{N}\left[a_{n} \sin \left(n \omega_{0} t\right)+b_{n} \cos \left(n \omega_{0} t\right)\right]+c_{1} g_{1}(t)
$$

where $c_{1}$ is one additional undetermined coefficient. Here, $g_{1}(t)$ is a function in the $\mathbf{C}^{1}$ function space of the exact solution as given, for example, by

$$
g_{1}(t)= \begin{cases}\left(t_{1}-T\right)^{3} t, & 0 \leqslant t<t_{1} \\ -2 T t^{3}+3 T\left(t_{1}+T\right) t^{2}+\left[2 t_{1}^{3}-\left(t_{1}+T\right)^{3}\right] t-t_{1}^{2} T\left(t_{1}-3 T\right), & t_{1} \leqslant t<T .\end{cases}
$$

Note that this function is periodic and $\mathbf{C}^{1}$ as defined by the conditions, $g_{1}(0)=g_{1}(T), \dot{g}_{1}(0)=$ $\dot{g}_{1}(T), g_{1}\left(t_{1}^{-}\right)=g_{1}\left(t_{1}^{+}\right)$, and $\dot{g}_{1}\left(t_{1}^{-}\right)=\dot{g}_{1}\left(t_{1}^{+}\right)$.

Substituting Eq. (11) into Eq. (1) leads to a new residual denoted $R_{2}(t)$ given by

$$
R_{2}(t)=R_{1}(t)+ \begin{cases}0, & 0 \leqslant t<t_{1} ; \\ c_{1} \ddot{g}_{1}(t)+\omega_{n}^{2} c_{1} g_{1}(t), & t_{1} \leqslant t<T .\end{cases}
$$


Here, the additional term in Eq. (11) is cancelled after substituting into Eq. (2) for the stick period $0 \leqslant t<t_{1}$ and only makes effects on the responses for the slip period $t_{1} \leqslant t<T$. Also, two times differention of the additional term is equal to zero for the stick period.

The additional d.o.f. added to the series now requires the additional orthogonality condition

$$
\left\langle R_{2}(t), g_{1}(t)\right\rangle=0 .
$$

The other orthogonality conditions and the initial conditions are exactly analogous to Eqs. (5)(7), (9), and (10) upon replacing $R_{1}(t)$ with $R_{2}(t)$ including $g_{1}(t)$ in the expansion. The resulting set of $2 N+4$ non-linear algebraic equations are again solved using Newton-Raphson.

\section{3. $\mathbf{C}^{1}$ expansion}

In this section, a new approximate series solution is sought whose terms have precisely the same degree of continuity as the exact solutions, namely $\mathbf{C}^{1}$. To construct this series, we introduce a discontinuous polynomial series expansion for the discontinuous acceleration

$$
\ddot{x}(t)=H\left(t-t_{1}\right)\left[\sum_{n=0}^{N} d_{n}\left(t-t_{1}\right)^{n}\right]
$$

where $H\left(t-t_{1}\right)$ is the Heaviside function and $t_{1}$ is the unknown transition time. Note that for $t<t_{1}, \ddot{x}=0$ and stick is captured exactly. Integrating Eq. (15) twice leads to the required $\mathbf{C}^{1}$ series expansion for the displacement

$$
x(t)=H\left(t-t_{1}\right)\left[\sum_{n=0}^{N} \frac{d_{n}}{(n+1)(n+2)}\left(t-t_{1}\right)^{n+2}\right]+e_{0} t+e_{1},
$$

where $e_{0}$ and $e_{1}$ are two constants of integration to be evaluated from the initial conditions.

Before proceeding further, it is worthwhile to discuss how the critical step above can be generalized to other examples. From the equations of motion, the approximate solution is selected to possess the same degree of continuity as the exact solution either in space or in time domains. Moreover, the degree of continuity can be determined by inspection of the equation(s) of motion. For example, the discontinuity first appears at the second spatial derivative of the displacement for a simply supported Euler-Bernoulli beam with a rotational spring in the interior [10]. Hence, the exact eigensolutions lie in $\mathbf{C}^{1}$ space. The approximate eigenfunctions (in space) are then constructed by first introducing a discontinuity in the second spatial derivative and then integrating twice to obtain the displacement. This procedure ensures that both approximate and exact solutions are in the same function space.

This idea can be readily applied to more examples, a few of which are briefly noted here. If a linear spring is attached to the beam instead of the rotational spring in the previous example, the eigensolutions are $\mathbf{C}^{2}$ as the spring produces a discontinuity in the internal shear force. When a discrete mass is attached to the interior of a string, cable, or rod, the eigensolutions are $\mathbf{C}^{0}$ due to the kink that may develop at the attached mass. If an impact is applied to the simple mass-spring oscillator, then the displacement is $\mathbf{C}^{0}$ (in time) and the velocity is discontinuous. By first noting the degree of continuity, the approximate solution can then be constructed in a fashion similar to that used in the friction oscillator detailed above. We now return to this example. 
Substituting Eq. (16) into Eq. (1) results in the residual $R_{3}(t)$ given by

$$
R_{3}(t)=\omega_{n}^{2}\left(e_{0} t+e_{1}\right)+H\left(t-t_{1}\right)\left\{\sum_{n=0}^{N} d_{n}\left(t-t_{1}\right)^{n}\left[1+\frac{\omega_{n}^{2}\left(t-t_{1}\right)^{2}}{(n+1)(n+2)}\right]\right\}-\frac{F_{f}}{M}
$$

Note that the obtained residual is equal to zero for the stick period since the approximate solution for $0 \leqslant t<t_{1}$ is equal to the exact solution.

The orthogonality conditions now require

$$
\left\langle R_{3}(t), \frac{H\left(t-t_{1}\right)\left(t-t_{1}\right)^{n+2}}{(n+1)(n+2)}\right\rangle=0, \quad n=0, \ldots, N .
$$

The integration constants are determined by inspection as $e_{0}=\dot{x}_{0}$ and $e_{1}=x_{0}$. Note that Eq. (18) leads $N+1$ coupled equations and the $N+3$ unknowns, $t_{1}, T$, and $d_{n}, n=0,1, \ldots, N$. The two additional equations,

$$
\begin{gathered}
x(t)=x(t+T) \Rightarrow \sum_{n=0}^{N}\left[\frac{d_{n}}{(n+1)(n+2)}\left(T-t_{1}\right)^{n+2}\right]+\dot{x}_{0} T=0, \\
\dot{x}(t)=\dot{x}(t+T) \Rightarrow \sum_{n=0}^{N}\left[\frac{d_{n}}{(n+1)}\left(T-t_{1}\right)^{n+1}\right]=0,
\end{gathered}
$$

enforce solution periodicity. The resulting set of $N+3$ equations are again solved by NewtonRaphson.

\section{Results}

An example system is studied herein as defined by the parameter values $M=10 \mathrm{~kg}, K=$ $1000 \mathrm{~N} / \mathrm{m}, V=1 \mathrm{~m} / \mathrm{s}, \mu_{s}=1 / 2$, and $\mu_{k}=1 / 4$. This example is now used to compare the three approximate solutions of Section 3 with the known exact solution.

Fig. 3 illustrates the computed approximate acceleration of the particle as a function of time (normalized with respect to the period $T$ ) using traditional harmonic balance. The approximate solution for $N=40$ (dashed curve) shows the expected oscillatory behavior (Gibbs phenomenon) near the discontinuity and converges to the arithmetic mean of the values on either side of the discontinuity as required. Hence, the series expansion converges to the exact solution (solid curve) in the mean square sense $\left(\mathbf{L}_{2}\right.$ norm).

The slow convergence of the classical harmonic balance method implied by the results of Fig. 3 is clearly observable in Fig. 4. This figure illustrates the estimated period $T$ of the periodic motion using the classical harmonic balance method $(\diamond)$, that method augmented by one $\mathbf{C}^{1}$ function $(\square)$, and the new $\mathbf{C}^{1}$ series (o). The classical harmonic balance series exhibits very slow convergence. The addition of a single $\mathbf{C}^{1}$ function dramatically improves the convergence rate. The expansion in purely $\mathbf{C}^{1}$ functions ultimately leads to even swifter convergence beyond $N=3$. Moreover, the estimated period using the new $\mathbf{C}^{1}$ series exhibits monotonic convergence beyond $N=3$ where it also remains within $\pm 0.1 \%$ of the exact period. 


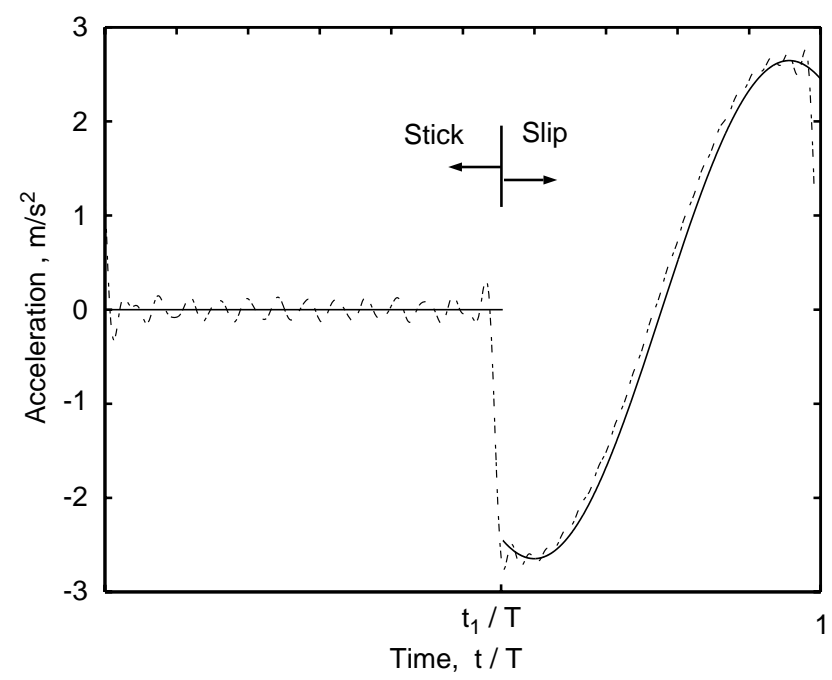

Fig. 3. Particle acceleration computed using the classical harmonic balance method (- - ) over one period $(N=40)$ and the exact solution $(-)$.

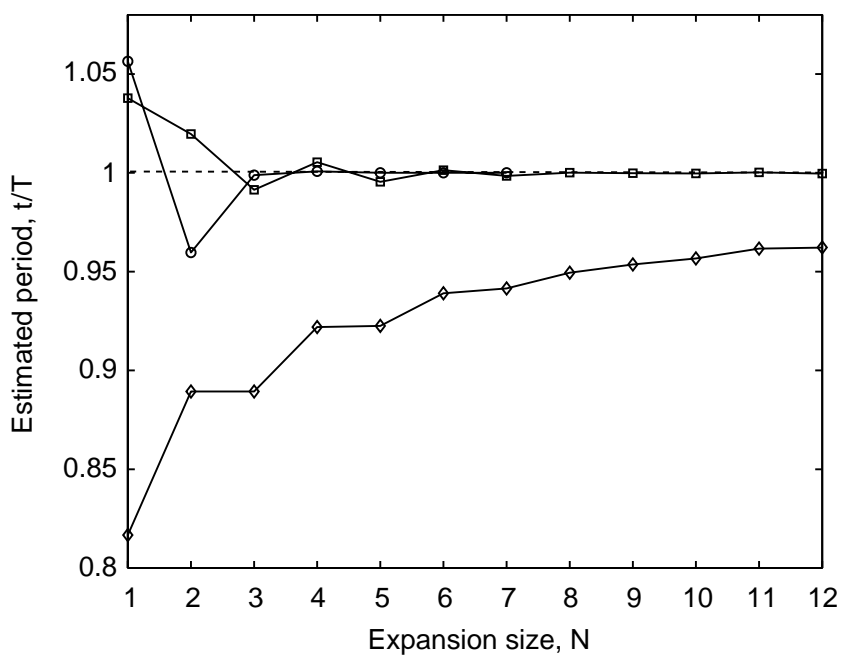

Fig. 4. Convergence of the computed period with expansion size $N$ : classical harmonic balance series $(\diamond)$, harmonic balance series augmented by one $\mathbf{C}^{1}$ function $(\square)$, and new $\mathbf{C}^{1}$ series ( $(\circ)$. The estimated period is normalized with the period of the exact solution (- - -).

Fig. 5 compares the computed acceleration response over one period for a comparable size series for all three methods $(N=3)$ together with the exact acceleration (solid curve). The classical harmonic balance solution (dash-dot curve) is relatively poor compared to the other approximations. Note that the Gibbs phenomenon present in the classical harmonic balance solution is entirely eliminated upon the addition of a single $\mathbf{C}^{1}$ term (dashed curve) (as well as in 


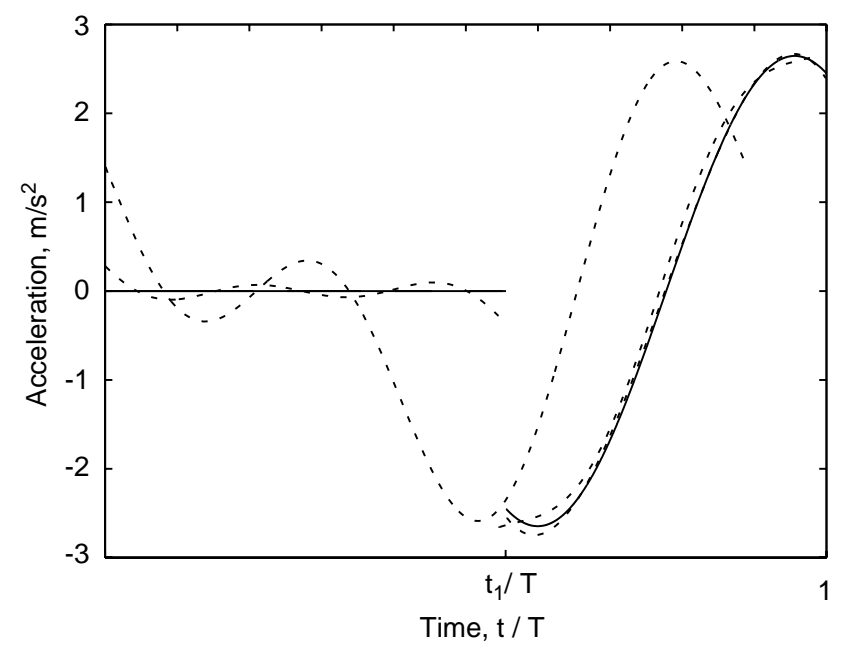

Fig. 5. Particle acceleration over one period: exact solution (-), classical harmonic balance (---), harmonic balance augmented by one $\mathbf{C}^{1}$ function (--), and new $\mathbf{C}^{1}$ series $(\cdots)$. In all cases, $N=3$.

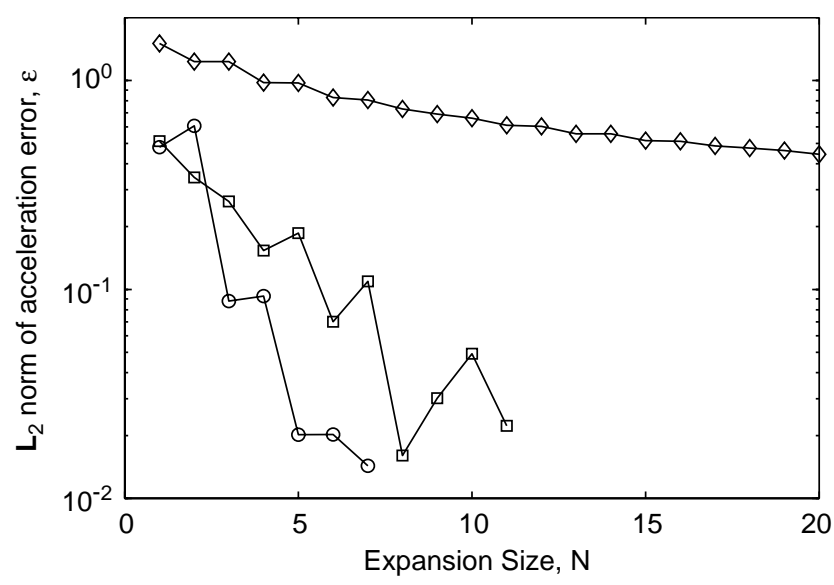

Fig. 6. $\mathbf{L}_{2}$ norm of acceleration error as a function of expansion size: classical harmonic balance $(\diamond)$, harmonic balance augmented by one $\mathbf{C}^{1}$ function ( $\square$ ), and new $\mathbf{C}^{1}$ series ( $($ ).

the new $\mathbf{C}^{1}$ series (dotted curve)). Moreover, both of these two latter series converge pointwise to the exact acceleration, whereas the classical harmonic balance solution converges only in the mean square sense.

The results of Fig. $5(N=3)$ are now extended in Fig. 6, where the $\mathbf{L}_{2}$ norm of the acceleration error is reported as a function of expansion size. Here, the $\mathbf{L}_{2}$ norm of a function $f$ (over one period),

$$
\|f\|=\sqrt{\int_{0}^{T} f^{2}(t) \mathrm{d} t}
$$


is used to compute the norm of the difference between an approximate solution and the exact solution for the acceleration, as defined by

$$
\varepsilon=\frac{\left\|\ddot{x}_{\text {exact }}-\ddot{x}_{\text {approx. }}\right\|}{\left\|\ddot{x}_{\text {exact }}\right\|} \text {. }
$$

This error measure for the acceleration using the classical harmonic balance $(\diamond)$ slowly decreases monotonically with increasing expansion size. However, the error for the classical harmonic balance augmented with one $\mathbf{C}^{1}$ term $(\square)$ decreases at a significantly greater rate (note log scale) although not monotonically. It is speculated that the non-monotonic behavior may occur because $g_{1}(t)$ may not be linearly independent of the Fourier series components of the expansion. For the new $\mathbf{C}^{1}$ series ( ${ }^{\circ}$ ), convergence is even swifter and resembles a stair-step pattern. This is expected since the even-order terms $(N=2,4, \ldots)$ are not expected to contribute to the antisymmetric acceleration response. Moreover, the new $\mathbf{C}^{1}$ series produces the swiftest convergence rate since all terms in the series are in the same function space as the exact solution.

\section{Summary and conclusion}

This paper presents generalizations of the harmonic balance/Galerkin method that substantially accelerate convergence relative to traditional harmonic balance for non-smooth dynamical systems. The methods follow from noting that the exact solution $x(t)$ of a non-smooth dynamical system possesses bounded continuity, $x(t) \in \mathbf{C}^{n}$, where $n$ is finite and can be deduced by inspection of the equation(s) of motion. Use of one or more expansion functions that lie in the function space $\mathbf{C}^{n}$ leads to the accelerated convergence observed herein. Moreover, the series convergence mode changes from that of mean square convergence to pointwise convergence for the discontinuous variable (for instance, the discontinuous acceleration in the case of a system with dry friction), and the Gibbs phenomenon is eliminated. Such methods hold promise for finding approximate periodic solutions of systems with dry-friction, impact, clearance, backlash, and other causes of non-smooth dynamic response.

\section{Acknowledgements}

The authors wish to acknowledge the US Office of Naval Research for support of this research.

\section{References}

[1] A.H. Nayfeh, D.T. Mook, Non-linear Oscillations, Wiley, New York, 1979.

[2] V.N. Pilipchuk, Analytical study of vibration systems with strong non-linearities by employing saw-tooth time transformations, Journal of Sound and Vibration 192 (1996) 43-64.

[3] V.N. Pilipchuk, Application of special nonsmooth temporal transformations to linear and non-linear systems under discontinuous and impulsive excitation, Non-linear Dynamics 18 (1999) 203-234.

[4] G. Salenger, A.F. Vakakis, Discreteness effects in the forced dynamics of a string on a periodic array of non-linear supports, International Journal of Non-linear Mechanics 33 (1998) 659-673.

[5] S. Wiggins, Introduction to Applied Non-linear Dynamical Systems and Chaos, Springer, New York, 1990. 
[6] Z.-R Liu, Discontinuous and impulsive excitation, Applied Mathematics and Mechanics 8 (1987) 31-35 (English edition).

[7] W. Szemplińska-Stupnicka, The Behavior of Nonlinear Vibrating Systems, Vol. I, Kluwer Academic Publishers, Dordrecht, 1990.

[8] I. Stakgold, Green's Functions and Boundary Value Problems, Wiley, New York, 1979.

[9] S.S.K. Tadikonda, H. Baruh, Gibbs phenomenon in structural mechanics, American Institute of Aeronautics and Astronautics Journal 29 (1991) 1488-1497.

[10] K. Gu, B.H. Tongue, A method to improve the modal convergence for structures with external forcing, American Society of Mechanical Engineers, Journal of Applied Mechanics 54 (1987) 904-909.

[11] H.P. Lin, N.C. Perkins, Free vibration of complex cable/mass systems: theory and experiment, Journal of Sound and Vibration 179 (1995) 131-149.

[12] M.-H.H. Shen, C. Pierre, Natural modes of Bernoulli-Euler beams with symmetric cracks, Journal of Sound and Vibration 138 (1990) 115-134.

[13] J.P. Den Hartog, Forced vibrations with combined Coulomb and viscous friction, Transactions of American Society of Mechanical Engineers 53 (1931) 107-115.

[14] R.A. Ibrahim, Friction-induced vibration, chatter, squeal, and chaos: Part I - mechanics of friction, American Society of Mechanical Engineers Winter Annual Meeting, California DE-49, 1992, pp. 107-121.

[15] R.A. Ibrahim, Friction-induced vibration, chatter, squeal, and chaos: Part II - dynamics and modeling, American Society of Mechanical Engineers Winter Annual Meeting, California DE-49, 1992, pp. 123-138.

[16] B. Feeny, A. Guran, N. Hinrichs, K. Popp, A historical review on dry friction and stick-slip phenomena, American Society of Mechanical Engineers Applied Mechanics Reviews 51 (1998) 321-341.

[17] S.W. Shaw, On the dynamic response of a system with dry friction, Journal of Sound and Vibration 108 (1986) 305-325.

[18] K. Popp, P. Stelter, Stick-slip vibrations and chaos, Philosophical Transactions of the Royal Society of London A332 (1990) 89-105. 